\title{
Anti-thrombotic effects of a-linolenic acid isolated from Zanthoxylum bungeanum Maxim seeds
}

\author{
Qian Yang ${ }^{1+}$, Weidong $\mathrm{Cao}^{2+}$, Xuanxuan Zhou', Wei Cao ${ }^{1}$, Yanhua Xie ${ }^{1 *}$ and Siwang Wang ${ }^{1 *}$
}

\begin{abstract}
Background: The current study was to evaluate the anti-thrombotic effect of alpha-linolenic acid (ALA) which was isolated and purified from Jiaomu in vivo.

Methods: The seeds were crushed and subsequently subjected to saponification, acid hydrolysis, gradient freezing, urea inclusion and complexation of silver nitrate to obtain the unsaturated fatty acids. The chemical characteristics of isolated ALA were validated by ${ }^{1} \mathrm{HNMR},{ }^{13} \mathrm{CNMR}$ and mass spectrometry, and then the anti-thrombotic effect of ALA and its mixture with linoleic acid (1:1) were evaluated in the following experiments.

Results: The alpha-linolenic acid was isolated and purified from Jiaomu through our newly established methods. ALA and its mixture with linoleic acid can prolong the hemorrhage and coagulation time as well as enhanced the survival rate of mice subjected to collagen-adrenaline induced thrombosis. In addition, the thrombosis on A-V bypass and platelet aggregation of rats will be reduced after treated with ALA or its mixture, and the expression level of Akt and PI3K protein decreased $26 \%$ and $31 \%$, respectively.

Conclusions: We designed and optimized a very simple and high-yield procedure to isolate ALA and linoleic acid mixture from seeds of Zanthoxylum bungeanum Maxim and demonstrated that such mixture can obtain a good anti-thrombotic effect through the modulation of PI3K/Akt signaling.
\end{abstract}

Keywords: Alpha-linolenic acid, Anti-thrombotic, Zanthoxylum bungeanum Maxim seeds, Linoleic acid, Unsaturated fatty acids

\section{Background}

Alpha-linolenic acid (ALA), which belongs to a group of fatty acids named omega-3 fatty acids, is an essential fatty acid. Seed oils are the richest sources of ALA, especially those of rapeseed, soybeans, walnuts, flaxseed, perilla, chia and hemp. ALA can be obtained from thylakoid membranes of the green leaves of broadleaf plants [1,2]. Similar to other essential fatty acids, ALA is critical for human bodily function given its conversion into the longer chain fatty acids eicosapentaenoic acid (EPA) and docosahexaenoic acid (DHA). ALA and its metabolic products, has long been known to reduce the risk of detrimental disorders including cardiac arrhythmia, high cholesterolemia, hypertension, thrombosis, allergy and cancer [3,4]. It was reported that ALA may possess the anti-arrhythmic properties after

\footnotetext{
*Correspondence: yhxiefmmu@163.com; siwwang@126.com

${ }^{\dagger}$ Equal contributors

'Department of Materia Medica, School of Pharmacology, Fourth Military Medical University, Xi'an, Shaanxi, China

Full list of author information is available at the end of the article
}

investigation of the association between arrhythmia and dietary ALA intake accessed via updated food-frequency questionnaires [5]. Intake of omega-3 fatty acids reduces risk of fatal coronary heart events, perhaps by stabilizing myocardium and reducing risk of fatal arrhythmias [6]. The wide variety of ALA-exerted biological actions may have relevance to their involvements in several physiological and pathological processes. ALA defect is closely related to the prevalence of hypertension, diabetes mellitus, coronary heart disease, schizophrenia, Alzheimer's disease, atherosclerosis and cancer, thus depicting the importance of adequate intake of ALA in daily life [7-9].

In spite of its crucial role in human health, dietary insufficiency in ALA is a rather common medical problem worldwide. As such, Food and Agriculture Organization of the United Nations and World Health Organization issued a joint statement, urging the necessity of increasing intake of ALA. Its annual demand has been over two million kilogram. Given ALA synthesis is still beyond 
our capability, it is of great importance to seek the natural sources of ALA.

Jiaomu (Semen Zanthozyli bungeani) is the seeds of Zanthoxylum bungeanum Maxim [10]. In the 1990s, groups of Chinese scientists discovered that the content of ALA in Jiaomu is about $17 \sim 24 \%$ [11,12], solidifying Jiaomu as a rich source of ALA. Being seeds of Zanthoxylum bungeanum Maxim, a traditional Chinese cuisine agent, 120 million kilogram of Jiaomu are discarded in China each year. As such, Jiaomu could be a rich source of ALA as long as the obstacles in its chemical production can be rectified. Up to date, highly efficient procedure is still lacked for the isolation and purification of ALA.

The aggregation and activation of platelet can affect the development of myocardial infarction, stroke and unstable angina, and thus, the anti-platelet agents can be induced the obviously antithrombotic effect and used to prevent various cardiovascular disorders. Furthermore, it has been demonstrated that GP IIb/IIIa receptor which can regulate the process of hemostasis in the body and be involved in the thrombus formation and platelet adhesion. The clinical application of platelet glycoprotein $I I b /$ IIIa (GP IIb/IIIa) antagonists can prevent the fibrinogenmediated aggregation of activated platelets [13-15]. But so far, the detail and further underlying mechanism on the modulation of anti-thrombotic process was still not clear after ALA treatment.

In the current study, firstly, we attempted to design and optimize the chemical strategy in the extraction of ALA from raw Jiaomu and evaluated its anti-thrombotic effect in vivo. In addition, the underlying mechanism of above process was also investigated to evaluate the future application of Jiaomu in health and dietary areas.

\section{Methods \\ Materials}

Authenticated, quality-certified raw Jiaomu were purchased from the Material Company of Hancheng (Shan'xi Province, China). Standard ALA compound was obtained from the Sigma Chemical Company (St Louis, MO, USA). It was dissolved in normal saline containing $0.5 \%$ Tween80 prior to use. Aspirin enteric-coated tablet was purchased from Bailu Pharm Co. Ltd., (Xian, China). Other analytical reagents were obtained from the Chemical Agent Factory of Xi'an (Xi'an, China).

All mice weighing at $18 \sim 22 \mathrm{~g}$ and rats weighing at $250 \sim 300$ g were provided by the animal center of Fourth Military Medical University. Animals were housed two per cage in a room controlled for temperature, lighting and humidity. All experiments were performed according to the ethical guidelines on animal care and were approved by the institutional animal care committee of Fourth Military Medical University.

\section{Preparation of ALA}

\section{Acid hydrolyze saponification}

The seeds were crushed and sifted out (40 60 mesh). The powder was decocted with $5 \% \mathrm{NaOH}$ for one hour at $65^{\circ} \mathrm{C}$ and filtrated to obtain the aqua part. Above process was repeated twice to combine the resulted filtrate. The solution was treated with $\mathrm{H}_{2} \mathrm{SO}_{4}$ (v/v, 1:1) to acidify to $\mathrm{pH}$ 2.0. The oil layer was separated and washed with water to $\mathrm{pH} 7.0$ to get the mixtures of fatty acid.

\section{Gradient freezing}

The mixtures of fatty acid mixed with $95 \% \mathrm{NaOH}(\mathrm{v} / \mathrm{v}$, 1:1) were placed at $0^{\circ} \mathrm{C}$ for $6 \sim 7 \mathrm{~h}$ before filtration. Then the solution was continuously frozen at $-5^{\circ} \mathrm{C}$ for $6 \sim 8 \mathrm{~h}$, filtrated and washed off residues with chilled $95 \% \mathrm{NaOH}$. The solvent was distilled-off to obtain the lipid portion.

\section{Urea inclusion}

The urea was dissolved into $95 \% \mathrm{NaOH}$, and then added into the frozen mixtures of the fatty acid (oil: urea $=1: 1$ ). After a sufficient mixing, the mixture was subsequently cooled at $3 \sim 5^{\circ} \mathrm{C},-6^{\circ} \mathrm{C}$ and $-12^{\circ} \mathrm{C}$ for $6 \sim 8 \mathrm{~h}$, and then quickly filtrated. The residue was washed off with $95 \%$ $\mathrm{NaOH}$ to obtain the oil after removing the solvent. The latter was bathed in water for 3 times, extracted with petrol ether, shaken with dried $\mathrm{MgSO}_{4}$ for $5 \sim 10 \mathrm{~min}$, and then filtrated. After contraction, the obtained extracts contained mostly unsaturated fatty acid (50\% ALA and $50 \%$ linoleic acid).

\section{Complexation of silver nitrate}

The mixtures of fatty acids were added into urea, mixed with $\mathrm{AgNO}_{3}$ solution at low temperature, stirred and then delaminated. The $\mathrm{AgNO}_{3}$ layer was partitioned in petrol ether and repeated $2 \sim 3$ times. The petrol ether layers were combined and concentrated to yield ALA.

\section{Chemical analysis}

The content of yielded ALA was analyzed by highperformance liquid chromatography (HPLC; Waters, Milford) using a C18 column $(4.6 \times 250 \mathrm{~mm} ; 5 \mu \mathrm{M})$ in 90:10 (v/v) acetonitrile/ $1 \%$ acetic acid solution delivered at $1 \mathrm{ml} / \mathrm{min}$ as the mobile phase. Elution of compounds was detected at $205 \mathrm{~nm}$ with $2996 \mathrm{UV}$ detector. ${ }^{1} \mathrm{HNMR}$,

${ }^{13} \mathrm{CNMR}$ and Mass spectrometry measurements were also performed to validate the produced compound, using commercial standard ALA compound as the control.

\section{Hemorrhage time and coagulation time determination}

All mice were randomly divided into the treated group with different dosage of ALA, negative (saline) and positive (aspirin) control groups. ALA treatment groups were received either pure ALA or its mixture with linoleic acid (ALA: linoleic acid $=1: 1)$ at 50, 100, or $250 \mathrm{mg}$ / 
$\mathrm{kg}$ for 10 days. Following the last administration, plateletrich plasma (PRP) was prepared and obtained from blood samples according to the previous methods [16,17]. Brifely, the sample containing $3.8 \%$ sodium citrate were centrifuged at $100 \times \mathrm{g}$ for $10 \mathrm{~min}$ at room temperature to obtain the PRP in supernatant. After that, the hemorrhage and coagulation time were determined by the related Kit ( $P T$ and $A P T T$ Kit) and Analyzer (LG-PABER, China).

\section{Thrombus formation analysis}

Mice were grouped and treated as described above. After the last treatment, a mixture of collagen $(3.57 \mathrm{mg} / \mathrm{kg})$ and adrenaline $(0.143 \mathrm{mg} / \mathrm{kg})$ were injected into vena caudal vein of mouse to stimulate thrombus formation for $5 \mathrm{~min}$ through the effect of platelet activation [18]. Mortality was calculated to determine the survival rate.

\section{Artery-venous (A-V) bypass thrombosis and Platelets aggregation analysis}

Rats were randomly divided into the treated and control groups according to the above procedure. Pure ALA compound or its mixture with linoleic acid (ALA: linoleic acid $=1: 1$ ) were administered at 35,70 or $150 \mathrm{mg} / \mathrm{kg}$ for 10 days. A-V bypass thrombosis analysis was performed as previously described to determine the wet weight of thrombus [19].

Venous blood was collected by atraumatic venepuncture into vacuette tubes containing sodium citrate $(3.8 \%, \mathrm{v} / \mathrm{v})$ after anesthetized animals. Platelet-rich plasma (PRP) was prepared according to the above method using collagen I $(22.0 \mu \mathrm{g} / \mathrm{ml})$ as an inducer. Maximum aggregation rate and aggregation inhibition rate were determined [20].

\section{Effect of ALA on P-selectin and GP Ilb/llla expression on washed platelets}

Platelets were prepared as described above. P-selectin (CD62) and GP IIb/IIIa expression was measured using FITC-labeled antibody. Briefly [21], platelets were washed and centrifuged, and then blocked with 5\% BSA solution. The antibody of P-selectin (CD62) and GP IIb/IIIa were incubated with sample for $30 \mathrm{~min}$ at $4^{\circ} \mathrm{C}$ in the dark, respectively. Flow cytometry (FCM, Becton Dickinson, San Jose, CA, USA) was performed to detect the expression of P-selectin and GP IIb/IIIa.

\section{Western bolt analysis}

Western blotting procedure was performed to investigate the changes on related protein expression in the presence or absence of ALA. Briefly, platelets were prepared as described above. Protein was collected in the following buffer including $0.1 \mathrm{M} \mathrm{NaCl}, 0.01 \mathrm{M}$ Tris- $\mathrm{HCl}$ (pH 7.6), 1\% (w/v) Triton X-100, 1 mM EDTA (pH 8.0), $100 \mathrm{mg} / \mathrm{ml}$ PMSF, $1 \%$ (w/v) NP-40, and $1 \mu \mathrm{g} / \mathrm{ml}$ leupeptin. After centrifugation at $10,000 \times g$ for $30 \mathrm{~min}$ at $4^{\circ} \mathrm{C}$, the total protein was harvested and quantified by the BCA protein assay kit (Pierce, Rockford, IL, USA). After that, the protein was separated by SDS-PAGE and electrophoretically transferred onto a PVDF membrane according to the standard western blotting protocol. The membrane was incubated with a primary antibody against PI3K and Akt (1:1000; Cell Signaling Technology, Beverly, MA, USA) overnight at $4^{\circ} \mathrm{C}$ after blocking with $5 \%$ milk in PBST. The enhanced chemiluminescence system was used to detect the positive belt. The blot was also re-probed and corrected by staining with $\beta$-actin antibody (Sigma, St Louis, $M O, U S A)$.

\section{Statistical analysis}

Data are presented as means \pm SD. Differences between groups were evaluated by the student's t test. ANOVA was applied to examine the difference in survival rate. All analyses were performed utilizing the SPSS software package (Version 11.5, SPSS Inc., Chicago, IL). Differences were considered statistically significant at $\mathrm{p}<0.05$.

\section{Results}

\section{Chemical characteristics}

The characteristics of isolated and purified alpha-linolenic acid (ALA) from Jiaomu were evaluated through the following experiments. A Quattro Premier MS system ( $\mathrm{Wa}$ ters Corp., Milford, MA, USA) operating under Masslynx 4.1 software was performed using liquid chromatography/electrospray ionization tandem mass spectrometry equipped with an electrospray ionization interface used to generate positive ions $\left[\mathrm{M}^{-} \mathrm{H}^{+}\right]$for the determination of ALA. The positive ion mass spectra of ALA showed stable molecular ions at $m / z=279.6$ (Figure $1 b$ ). The source temperature was $110^{\circ} \mathrm{C}$. The electrospray capillary voltage was $3.0 \mathrm{kV}$, nitrogen was the desolvation gas ( $500 \mathrm{~mL} / \mathrm{min}$ flow rate). Compounds were separated on a reversed-phase column by a Waters 2695 HPLC system. The column temperature was $25^{\circ} \mathrm{C}$. The retention time of isolated ALA sample was $6.036 \mathrm{~min}$, identical to that of the standard compound (Figure 1d). The purity of ALA ranged from $91.6 \%$ to $95.2 \%$. NMR analysis data were presented as follows: ${ }^{13} \mathrm{CNMR} \delta /$ ppm (Figure 2a): $180.43\left(\mathrm{C}_{1}\right), 134.13\left(\mathrm{C}_{2}\right), 131.85\left(\mathrm{C}_{9}\right)$, $130.14\left(\mathrm{C}_{16}\right), 128.26\left(\mathrm{C}_{12}\right), 128.22\left(\mathrm{C}_{13}\right), 127.80\left(\mathrm{C}_{15}\right)$, $127.16\left(C_{10}\right), 29.62\left(C_{6}\right), 29.21\left(C_{7}\right), 29.13\left(C_{5}\right), 29.09$ $\left(\mathrm{C}_{4}\right), 27.23\left(\mathrm{C}_{8}\right), 25.65\left(\mathrm{C}_{14}\right), 25.56\left(\mathrm{C}_{11}\right), 24.69\left(\mathrm{C}_{3}\right)$, $20.56\left(\mathrm{C}_{17}\right), 14.26\left(\mathrm{C}_{18}\right) ;{ }^{1} H N M R \quad \delta / p p m$ (Figure $2 \mathrm{~b}$ ): 11.4 (br, s, $1 \mathrm{H},-\mathrm{COOH}), 5.3 \sim 5.4(\mathrm{~m}, 6 \mathrm{H},-\mathrm{CH}-\mathrm{CH}-$, $\left.\mathrm{C}_{9}, 10,12,13,15,16\right), 2.8$ (t, 4H, $\left.\mathrm{CH}_{2}, \mathrm{CH}_{2}, \mathrm{C}_{11},{ }_{14}\right), 2.35$ $\left(\mathrm{t}, 2 \mathrm{H}, \mathrm{CH}_{2}, \mathrm{C}_{2}\right), 2.06\left(\mathrm{~m}, 4 \mathrm{H}, \mathrm{CH}_{2}, \mathrm{C} 8,17\right), 1.63(\mathrm{~m}$, $\left.2 \mathrm{H}, \mathrm{CH}_{2}, \mathrm{C}_{3}\right), 1.32\left(\mathrm{~m}, 8 \mathrm{H}, \mathrm{CH}_{2}, \mathrm{C}_{4,5,6,7}\right), 0.98(\mathrm{t}, 3 \mathrm{H}$, $\mathrm{CH}_{3}, \mathrm{C}_{18}$ ). And above data also provided the certainly evidence for efficiently isolated and purified methods of ALA from Jiaomu. 


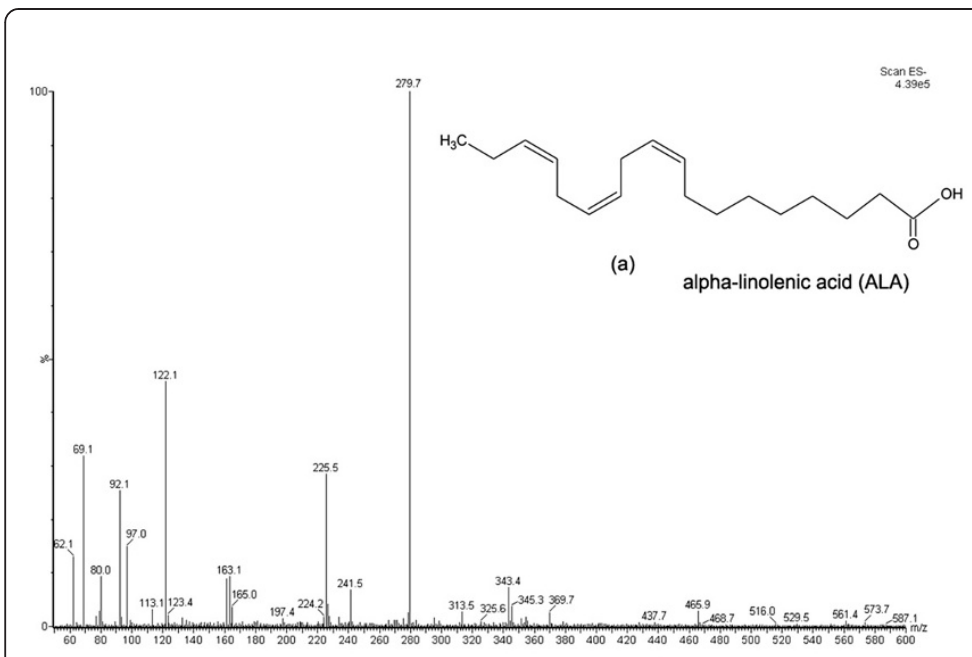

(b)

Figure 1 Chemical characteristics of alpha-linolenic acid. (a) Chemical structure of alpha-linolenic acid; (b) Negative ion mass spectra of alpha-linolenic acid, $\mathrm{m} / \mathrm{z}=279.6$; (c) UV; (d) HPLC.

\section{Effects of ALA on hemorrhage and coagulation time}

To explore the effect of ALA on the stanching and coagulation functions of mice, we examined the hemorrhage and coagulation time of ALA or its mixture treated mice. As shown in Figure 3a or b, pure ALA with lower, middle or higher dosage $(50,100$ or $250 \mathrm{mg} / \mathrm{kg}$ ) prolonged the hemorrhage and coagulation time, respectively. The mixture composed of ALA and linoleic acid (1:1) also remarkably increased hemorrhage and coagulation time. For above positive effects on hemorrhage time, there is no clear difference among each treated groups, but for coagulation time, the effects displayed with a concentration-dependent manner both in pure and mixture treated groups. In the group of mixture with higher dosage, the coagulation time increased at least 35\% compared with the saline treated group. Our results indicated that both ALA and the mixture treatment significantly inhibited blood standing and coagulation in mice.

\section{Effects of ALA on thrombosis}

We further determined the therapeutic effect of ALA and its mixture on stimulate thrombosis formation. Collagen and adrenaline efficiently induced thrombus formation in mice, as revealed by rapid mortality of most animals following the inducer treatment. Similar to aspirin, ALA enhanced the survival rate of the animals significantly (Figure 3c). After treated with $250 \mathrm{mg} / \mathrm{kg}$ ALA, the survival rate increased at least 3.5 times compared with saline treated group, indicating that ALA can completely inhibit collagen- and adrenaline-induced thrombosis in mice at this concentration. And for the mixture treated group with higher dosage, the similar effects were also observed and no difference between these two groups.
After treated with ALA or its mixture in rats, A-V bypass thrombosis was also evaluated in each group. Likewise, ALA and its mixture were found to inhibit collagen stimulated A-V bypass thrombosis in rats. Both pure ALA and mixture with lower, middle or high dosage $(35,70$ or $175 \mathrm{mg} / \mathrm{kg}$ ) suppressed A-V thrombus formation, respectively. Compared with saline treated group, the thrombotic wet weight decreased $33 \%$ and $42 \%$ after treated with ALA or its mixture group, respectively (Figure 3d). Our above results indicated that the ALA mixture offered a better therapeutic effect than the pure ALA compound.

\section{Effects of ALA on collagen-induced platelet aggregation in rats}

Moreover, we explored the effect of ALA and its mixture on the collagen-induced platelet aggregation (Figure 3e). Firstly, the effect of aspirin $(100 \mathrm{mg} / \mathrm{kg})$ on the inhibition of collagen stimulated platelet aggregation was observed in vitro. After treated with middle or high dosage (70 or $175 \mathrm{mg} / \mathrm{kg}$ ) pure ALA, such inhibition was also occurred obviously, the platelet aggregation decreased $29 \%$ or $34 \%$ in middle or higher dosage group, respectively. But for lower dosage $(35 \mathrm{mg} / \mathrm{kg})$ group, there was no significant changes compared with saline treated group. Similar results were also observed after treated with ALA mixture, the platelet aggregation decreased $39 \%$ or $46 \%$ in middle or high dosage group, respectively.

\section{Effect of ALA on P-selectin and GP IIb/IIla expression}

The effects on platelet P-selectin and GP IIb/IIIa expression were examined by FCM after treated with pure ALA or its mixture. As shown in Figure 4a, ALA reduced P-selectin secretion at $12 \%, 27 \%$ or $32 \%$ in the different group with lower, middle or higher dosage, respectively. 


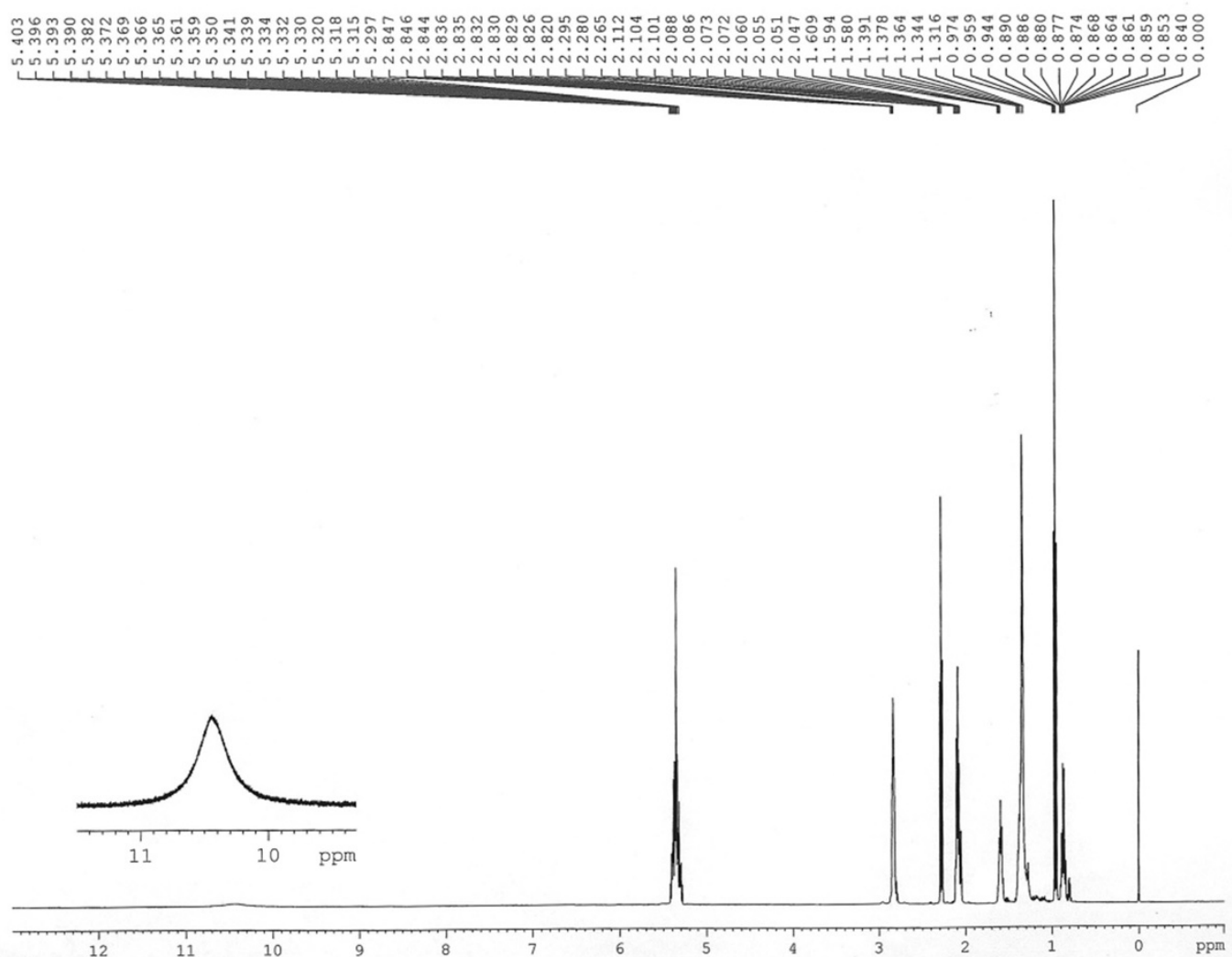

(a)
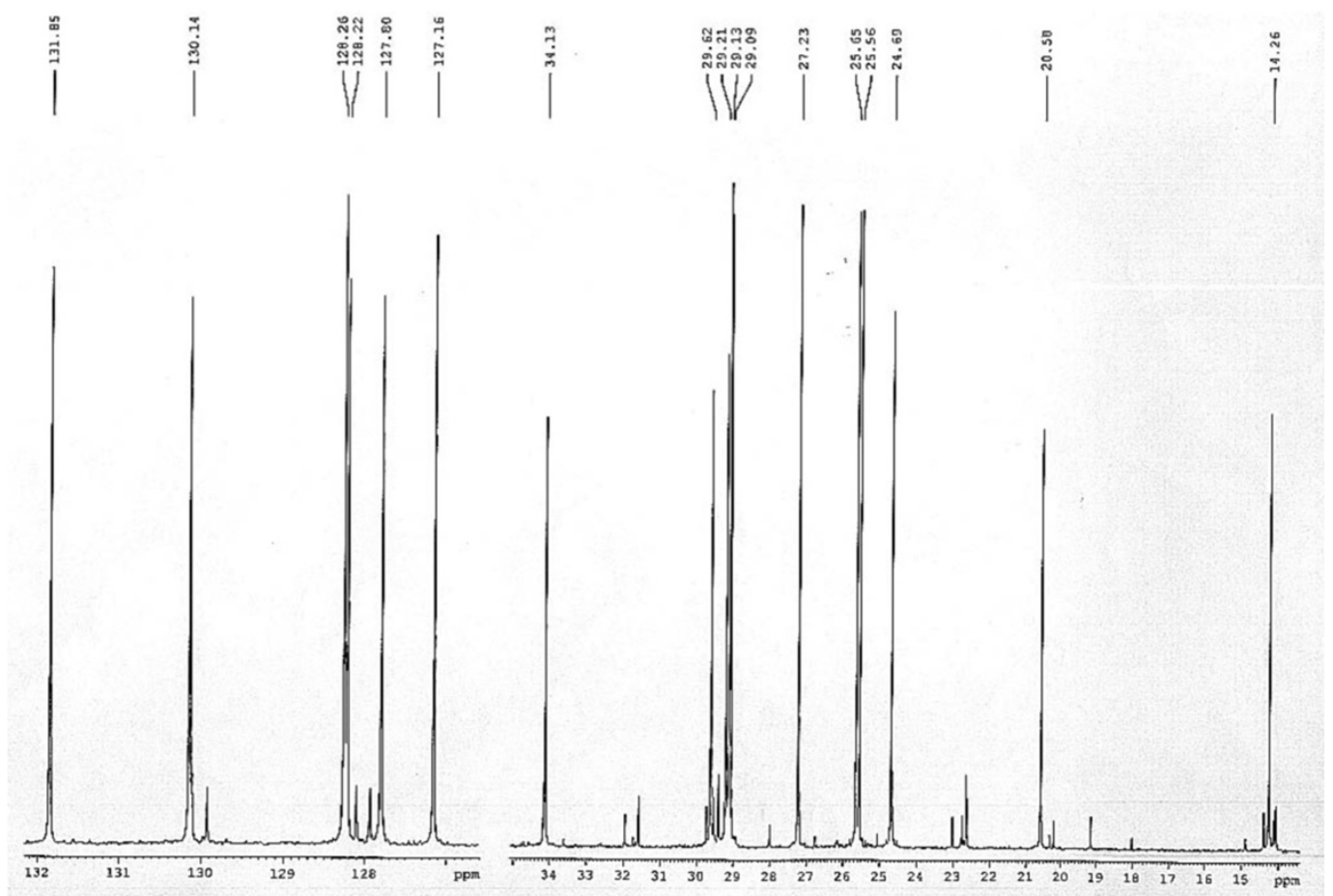

(b)

Figure 2 NMR analysis data of alpha-linolenic acid. (a) ${ }^{1} \mathrm{HNMR} \delta / \mathrm{ppm}$; (b) ${ }^{13} \mathrm{CNMR} \delta / \mathrm{ppm}$. 
a
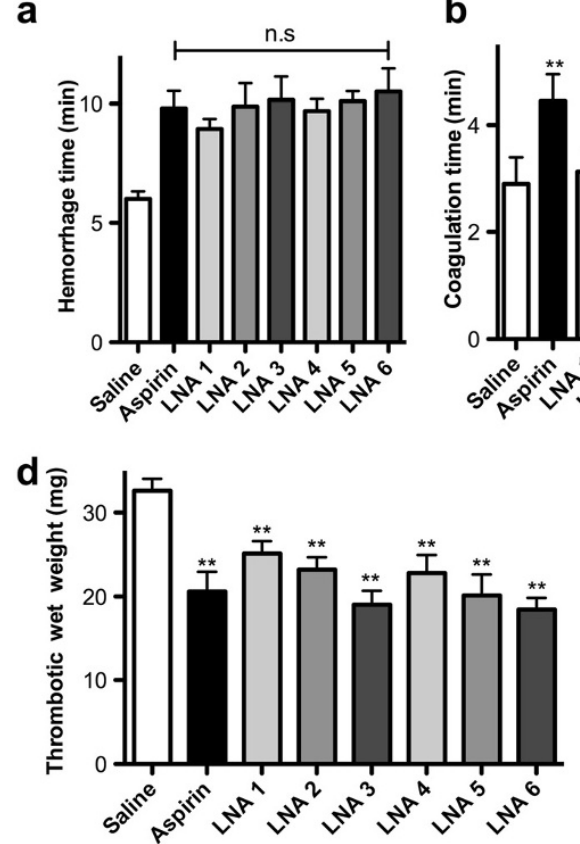

b

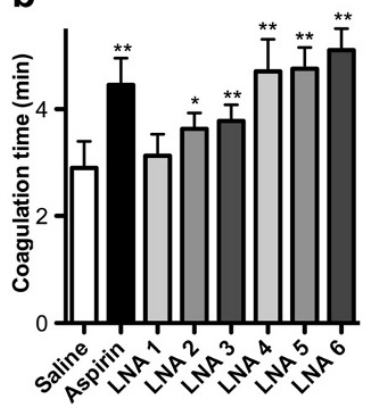

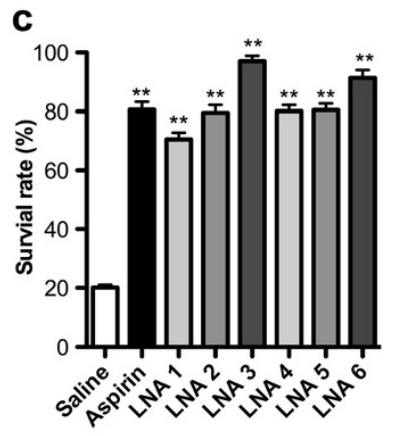

e

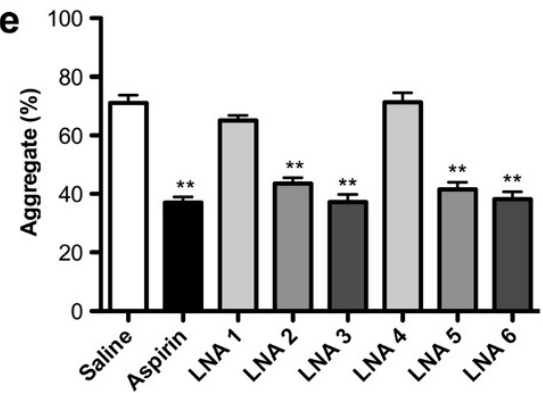

Figure 3 Anti-thrombotic effects of alpha-linolenic acid. (a) Effects of ALA on hemorrhage time; (b) Effects of ALA on coagulation time; (c) Effect of ALA on trombus formation analysis, mortality was calculated to determine the survival rate; (d) Effect of ALA on artery-venous (A-V) bypass thrombosis, the wet weight of thrombus was determined by subtracting the weight of dry cotton thread; (e) Rat platelets were isolated and platelet aggregation was determined in vitro. Aspirin (100 mg/kg), a-LNA 1-3 (pure ALA: 50, 100 and 250 mg/kg), a-LNA 4-6 (ALA mixture: 50, 100 and $250 \mathrm{mg} / \mathrm{kg}){ }^{*} \mathrm{p}<0.05,{ }^{* *} \mathrm{p}<0.01$ vs. control group.

And ALA mixture can reduce P-selectin secretion at 13\%, $36 \%$ or $41 \%$, respectively. In addition, the expression of GP IIb/IIIa decreased $25 \%, 31 \%$ or $35 \%$ in ALA treated group with lower, middle or higher dosage, respectively. Similar tendency on GPIIb-IIIa expression were observed after ALA mixture treatment, it decreased $24 \%, 38 \%$ or $45 \%$, respectively (Figure $4 \mathrm{~b}$ ).

\section{Effect of ALA on PI3K signaling}

The expression level of Akt and PI3K, which are directly related to changes in thrombosis formation process, were examined after ALA and its mixture treatment through western blot analysis. The results showed that Akt expression decreased 26\% in ALA treated group, and 35\% in ALA mixture treated group, respectively. After treated

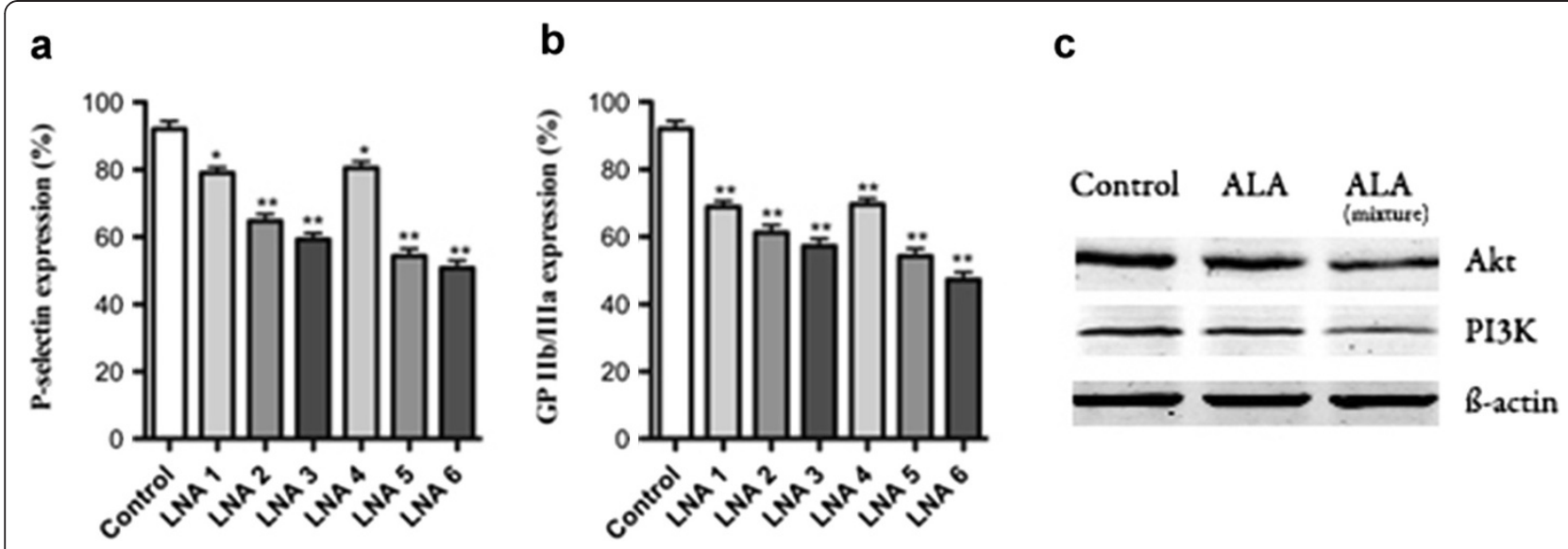

Figure 4 Effect of ALA on P-selectin, GP IIb/IIla expression and PI3K signaling. Effect of ALA on P-selectin (a) and GP /Ib/IIla (b) expression $\left({ }^{*} p<0.05,{ }^{* *} p<0.01\right.$ vs. control group.); (c) western blot results showed the effect of ALA on PI3K and AKT expression. a-LNA 1-3 (pure ALA: 50, 100 and $250 \mathrm{mg} / \mathrm{kg}$ ), a-LNA 4-6 (ALA mixture: 50, 100 and $250 \mathrm{mg} / \mathrm{kg}$ ). 
with ALA, the inhibition on Akt changes was clearly. Furthermore, the expression of PI3K were also decreased 31\% and 39\% after treated with ALA and its mixture, respectively (Figure 4). Above results provided the direct evidence that both ALA and its mixture have the obvious antithrombosis effects which were related to the changes of PI3K or Akt protein expression level, and such effects of ALA on the protein changes was similar with ALA mixture treated group, which was the possible reason for the above similar tendency on anti-thrombosis effects between ALA and its mixture.

\section{Discussion}

In the current study, our data provided the following evidences: (1) the highly purified ALA was isolated from Jiaomu through our newly established methods; (2) ALA and its mixture can reduce the thrombosis on A-V bypass and platelet aggregation; (3) The hemorrhage and coagulation time can be prolonged after treated with ALA and its mixture with linoleic acid; (4) ALA and its mixture application can reduce the P-selectin secretion and GPIIb-IIIa expression; (5) Anti-thrombotic effect of ALA was related to the expression of PI3K and Akt, which may be the possible mechanism of above process. And thus, above data demonstrate that ALA can affect the process of thrombotic through the modulation of PI3K/Akt signaling.

We designed and optimized the isolation procedure of ALA from Jiaomu, seeds of Zanthoxylum bungeanum Maxim, and demonstrated an important role of these seeds as a potential source of ALA. Given that most fatty acids exist along with lipid, we attempted to extract the fatty acid mixture by acid leaching following decoction in $5 \% \mathrm{NaOH}$. Our current approach was revealed to be more efficient than the traditional method in separating lipids from raw materials. Moreover, our method is more feasible for industrial production. Based on the various freezing points of fatty acids, we applied the gradient freezing method to facilitate removal of wax, saturated fatty acids, mono- and di-unsaturated fatty acids which should help to reduce consumption of urea and enhance its efficiency. As previously reported [22], urea can form stable inclusion with saturated and mono-unsaturated fatty acids at low temperature, which results in isolate polyunsaturated fatty acids. Since urea also form inclusion with some of unsaturated fatty acids, it is crucial to maintain proper urea content in order to prevent the loss of ALA.

It was demonstrated that the function of blood stanching and coagulation can be represented through the evaluation on hemorrhage and coagulation time. According to our results, ALA treatment prolonged both indices in mice, consistent with the results from previously report [23]. Furthermore, ALA isolated from the seeds can greatly enhance the survival rate of mice suffering from collagen/ adrenaline-induced thrombosis and decrease the mass of thrombus formed during A-V bypass. All these data collectively confirmed the in vivo activity of extracted ALA and thus demonstrated that the isolation procedure reported in our study not only increases the yield but also successfully maintains the biological activity of the compound of interest.

It has been evidenced that the process of platelet aggregation and thrombus formation was related to the P-selectin secretion and GP IIb/IIIa activation which was also crosslinked Akt activation [24,25]. Our current results provided the certainly evidence that ALA can reduce P-selectin secretion and GP IIb/IIIa expression obviously. Furthermore, the platelet function, including activation, adhesion, spreading, and aggregation, was related to the changes on the signaling pathway of PI3K and Akt which was the main target of PI3K [26,27]. Our results also showed that ALA reduced the expression level of PI3K and Akt. And such results demonstrated that the anti-thrombotic effect of ALA will be related to the PI3K signaling pathway.

In our current study, it was interested to find that the biological activity of ALA and linoleic acid (1:1) mixture is superior to that of the pure ALA. We speculated that it may be ascribed to the competition of linoleic acid with ALA in metabolism. Although the two acids have their specific metabolic pathways, they share the similar enzymes. ALA is metabolized into EPA and DHA by the action of a serious of desaturase and elongase, while linoleic acid is converted to arachidonic acid (AA). The competition between ALA and linoleic acid suppresses the production of AA, which competes with EPA for cyclooxygenase and lipooxidase and consequently inhibits the generation of leukotrienes and prostaglandins. According to our results, ALA and linoleic acid mixture at 1:1 can achieve a better anti-thrombosis efficiency than ALA alone, suggested that their relative ratio is crucial for the ultimate therapeutic effect. Notably, the urea inclusion method applied in the current study yielded the mixture, consisting of 50\% ALA and 50\% linoleic acid. The extracts from Jiaomu through urea inclusion cannot only achieve favorable therapeutic effect, but also greatly simplify the production procedure and costs.

\section{Conclusions}

In summary, we designed and optimized a very simple and high-yield procedure to isolate ALA and linoleic acid mixture from seeds of Zanthoxylum bungeanum Maxim. The data demonstrated that such mixture can achieve a better anti-thrombosis effect than pure ALA through the modulation of PI3K/Akt signaling. Our results favor the seeds as a rich source of ALA and consolidate its future application in dietary and medical areas.

\section{Competing interests}

The authors declare that they have no competing interests. 


\section{Authors' contributions}

QY, WDC and SW designed the study; QY, XZ, WC and YX performed the experiments; WDC and SW prepared the manuscript. All authors read and approved the final manuscript.

\section{Acknowledgements}

The authors thank Hua Li for his technical assistance.

\section{Author details}

'Department of Materia Medica, School of Pharmacology, Fourth Military Medical University, Xi'an, Shaanxi, China. ${ }^{2}$ Department of Neurosurgery, Xijing Hospital, Fourth Military Medical University, Xi'an, Shaanxi, China.

Received: 28 May 2014 Accepted: 16 September 2014

Published: 23 September 2014

\section{References}

1. Deckelbaum RJ, Torrejon C: The omega-3 fatty acid nutritional landscape: health benefits and sources. J Nutr 2012, 142:587S-591S.

2. Rao S, Abdel-Reheem M, Bhella R, McCracken C, Hildebrand D: Characteristics of high alpha-linolenic acid accumulation in seed oils. Lipids 2008, 43:749-755.

3. Arshad A, Al-Leswas D, Stephenson J, Metcalfe M, Dennison A: Potential applications of fish oils rich in $\mathrm{n}-3$ fatty acids in the palliative treatment of advanced pancreatic cancer. Br J Nutr 2011, 106:795-800.

4. Meijerink J, Balvers M, Witkamp R: $\mathrm{N}$-acyl amines of docosahexaenoic acid and other $\mathrm{n}-3$ polyunsatured fatty acids - from fishy endocannabinoids to potential leads. Br J Pharmacol 2013, 169:772-783.

5. Albert CM, Oh K, Whang W, Manson JE, Chae CU, Stampfer MJ, Willett WC, Hu FB: Dietary alpha-linolenic acid intake and risk of sudden cardiac death and coronary heart disease. Circulation 2005, 112:3232-3238.

6. Harris WS: Extending the cardiovascular benefits of omega-3 Fatty acids. Curr Atheroscler Rep 2005, 7:375-380.

7. Das UN: Essential fatty acids: biochemistry, physiology and pathology. Biotechnol J 2006, 1:420-439.

8. Pan A, Chen M, Chowdhury R, Wu JH, Sun Q, Campos H, Mozaffarian D, Hu FB: a-Linolenic acid and risk of cardiovascular disease: a systematic review and meta-analysis. Am J Clin Nutr 2012, 96:1262-1273.

9. Poudyal H, Panchal SK, Diwan V, Brown L: Omega-3 fatty acids and metabolic syndrome: effects and emerging mechanisms of action. Prog Lipid Res 2011, 50:372-387.

10. Li Y, Zeng J, Liu L, Jin X: GC-MS analysis of supercritical carbon dioxide extraction products from pericarp of Zanthoxylum bungeanum. Zhong Yao Cai 2001, 24:572-573.

11. Li GH, Fu LM, Xue KF: Examination of the chemical composition of the seeds of Zanthoxylum bungeeanum Maxim. J Zhengzhou Grain College 1994, 15:21-23.

12. Zhuang SH, Li ML: Ingredient analysis of prickly ash seed oil. Acta Agriculturae Boreali-occidentalis Sinica 2002, 11:43-45.

13. Hook KM, Bennett JS: Glycoprotein Ilb/llla antagonists. Handb Exp Pharmacol 2012, 210:199-223

14. Lippi G, Montagnana M, Danese E, Favaloro EJ, Franchini M: Glycoprotein Illb/lla inhibitors: an update on the mechanism of action and use of functional testing methods to assess antiplatelet efficacy. Biomark Med 2011, 5:63-70

15. Ciborowski M, Tomasiak M: The in vitro effect of eptifibatide, a glycoprotein $\mathrm{llb} / \mathrm{lll}$ la antagonist, on various responses of porcine blood platelets. Acta Pol Pharm 2009, 66:235-242.

16. Kisucka J, Chauhan AK, Zhao BQ, Patten IS, Yesilaltay A, Krieger M, Wagner DD: Elevated levels of soluble P-selectin in mice alter blood-brain barrier function, exacerbate stroke, and promote atherosclerosis. Blood 2009, 113:6015-6022.

17. Dona M, Fredman G, Schwab JM, Chiang N, Arita M, Goodarzi A, Cheng G, von Andrian UH, Serhan CN: Resolvin E1, an EPA-derived mediator in whole blood, selectively counterregulates leukocytes and platelets. Blood 2008, 112:848-855

18. Miszti-Blasius K, Debreceni IB, Felszeghy S, Dezso B, Kappelmayer J: Lack of P-selectin glycoprotein ligand-1 protects mice from thrombosis after collagen/epinephrine challenge. Thromb Res 2011, 127:228-234.

19. Umar A, Guerin V, Renard M, Boisseau M, Garreau C, Begaud B, Molimard M, Moore N: Effects of armagnac extracts on human platelet function in vitro and on rat arteriovenous shunt thrombosis in vivo. Thromb Res 2003, 110:135-140.

20. Chen G, Fei $X$, Ling J: The effects of aminoglycoside antibiotics on platelet aggregation and blood coagulation. Clin Appl Thromb Hemost 2012, 18:538-541.

21. Oh WJ, Endale M, Park SC, Cho JY, Rhee MH: Dual Roles of Quercetin in Platelets: Phosphoinositide-3-Kinase and MAP Kinases Inhibition, and CAMP-Dependent Vasodilator-Stimulated Phosphoprotein Stimulation. Evid Based Complement Alternat Med 2012, 2012:485262.

22. Zhang HM, Liu FZ, Dai LM: Parity method of a-linlenic acid based on the theory of urea addution fractionation(i)-study on the purification process with orthogonal experiment. China Oils Fats 2001, 26:41-44.

23. Renaud S, Lanzmann-Petithory D: Dietary fats and coronary heart disease pathogenesis. Curr Atheroscler Rep 2002, 4:419-424.

24. Moore SF, Hunter RW, Harper MT, Savage JS, Siddiq S, Westbury SK, Poole $A W$, Mumford AD, Hers I: Dysfunction of the PI3 kinase/Rap1/integrin $a(I l b) \beta(3)$ pathway underlies ex vivo platelet hypoactivity in essential thrombocythemia. Blood 2013, 121:1209-1219.

25. Schaff M, Receveur N, Bourdon C, Ohlmann P, Lanza F, Gachet C, Mangin $\mathrm{PH}: \beta$-arrestin-1 participates in thrombosis and regulates integrin allb $\beta 3$ signalling without affecting $P 2 Y$ receptors desensitisation and function. Thromb Haemost 2012, 107:735-748.

26. Park JY, Ji HD, Jeon BR, Im EJ, Son YM, Lee JY, Lee DH, Lee YC, Hyun E, Jia Q, Hong M, Park HJ, Rhee MH: Chlorin e6 Prevents ADP-Induced Platelet Aggregation by Decreasing PI3K-Akt Phosphorylation and Promoting cAMP Production. Evid Based Complement Alternat Med 2013, 2013:569160.

27. Hadas K, Randriamboavonjy V, Elgheznawy A, Mann A, Fleming I: Methylglyoxal induces platelet hyperaggregation and reduces thrombus stability by activating PKC and inhibiting PI3K/Akt pathway. PLoS One 2013, 8:e74401.

doi:10.1186/1472-6882-14-348

Cite this article as: Yang et al: Anti-thrombotic effects of a-linolenic acid isolated from Zanthoxylum bungeanum Maxim seeds. BMC Complementary and Alternative Medicine 2014 14:348.

\section{Submit your next manuscript to BioMed Central and take full advantage of:}

- Convenient online submission

- Thorough peer review

- No space constraints or color figure charges

- Immediate publication on acceptance

- Inclusion in PubMed, CAS, Scopus and Google Scholar

- Research which is freely available for redistribution 